\title{
A Prototype Fingertip with High Spatial Resolution Pressure Sensing for the Robot iCub
}

\author{
Alexander Schmitz ${ }^{1}$, Marco Maggiali ${ }^{1}$, Marco Randazzo ${ }^{1}$, Lorenzo Natale ${ }^{1}$ and Giorgio Metta ${ }^{1,2}$ \\ ${ }^{1}$ Italian Institute of Technology, Italy \\ ${ }^{2}$ DIST, University of Genoa, Italy \\ alexander.schmitz, marco.maggiali, marco.randazzo, lorenzo.natale,giorgio.metta@iit.it
}

\begin{abstract}
Tactile feedback is of crucial importance for object manipulation in unknown environments. In this paper we describe the design and realization of a fingertip which includes a capacitive pressure sensor with 12 sensitive zones. It is naturally shaped and its size is small enough so that it can be mounted on the fingers of the humanoid robot iCub. It also embeds the electronic device which performs A/D conversion: This is beneficial for the signal to noise ratio and reduces the number of wires required to connect the fingertip to the robot. The fingertip is made of silicone, which makes its surface and inner structure compliant and flexible. We present preliminary experiments performed with the first prototype.
\end{abstract}

\section{INTRODUCTION}

Robotic agents can manipulate objects quickly, precisely and reliably in controlled settings, such as industrial environments. However, they often fail to achieve such abilities in unknown environments, or with novel objects. Humans, on the other hand, are consistently skillful at such actions. One of the reasons why current robotic systems lag behind is that they lack accurate and precise tactile feedback.

Therefore, to achieve human-like object manipulation, a robotic hand not only needs a high degree of actuation (i.e. many degrees of freedom), but should be also equipped with sophisticated haptic sensors [1]. This sensory information is essential for adapting the grasp, and in addition can be used to actively explore the object in-hand. Thus, it is possible to obtain information about objects that is hard or even impossible to acquire through other sensing modalities such as vision or sound. This is important because grasping and object manipulation do not depend only on visible properties such as size, orientation and shape, but also, for example, on weight, slipperiness, texture and hardness. Even visible features, such as the shape and the edges of an object, are sometimes easier to detect with haptic sensors.

In general, object manipulation enables active object exploration and induces sensory-motor correlations. Many researchers see perception as an active process, where an agent actively structures its own sensory input by manipulating the world to obtain "good" sensory data, enabling categorization, adaptation and learning (see for example [2][3][4]).

Another important aspect when designing a robotic hand is compliance. On the one hand, planning movements accurately beforehand is often too hard, on the other feedback control is often too slow to affect control appropriately [5][6]. A number of researchers therefore have started to emphasize the role of passive compliance (for example through the use of elastic materials) for dealing with uncertainties [7][8][9][10]. Even in the absence of sophisticated control, passive compliance facilitates movements in unknown environments.

Therefore, passive compliance is an important basis for grasping. It has the benefit of immediate response without any time-delay and no computational effort. The grasping task is split up between the controller and the morphology of the robot. A compliant finger leads to the compensation of small errors in the configuration of the grasp. This allows, for example, faster grasping movements and a more robust handling of objects. It creates grasp adaptability and stability, and provides protection against impacts [11].

When the compliant material is attached on top of the sensory surface, the drawback is that it acts as a low pass filter which reduces the spatial resolution of the sensor [1]. It would be therefore beneficial if no material was mounted on top of the transducer, but instead the transducer was compliant, and additionally was also mountable on top of a flexible material.

A number of robotic hands for object manipulation have been built. For example, the 6-axis force sensor used in DLRHIT hand [12], the GIFU III hand [13] and the 3-axis force sensor in the Paloma hand [14] can measure pressure and shear forces. The GIFU III hand has 859 sensing points, however because of the nature of the sensors, the skin is not soft and the signal conditioning happens at a distance from the sensitive area. The Obrero hand [15] has 40 contact points which embeds 4 sensors each. The Shadow hand [16] has a high dexterity, comparable to that of the human hand. It also has a high number of sensors in its fingertips (based on QTC technology) and adjustable compliance in the actuation system which is pneumatic.

\section{The Hands of the Humanoid Robot ICub}

The fingertip described here will be mounted on the humanoid robot iCub [17] (see also Figure 1). iCub has a complete body with two legs, two arms, two hands and a head. It has 53 controllable degrees of freedom altogether, 9 of which belong to each hand, excluding the wrist. The robot is about $1 \mathrm{~m}$ tall, that is roughly the size of a three and a half years old child. It is equipped with an active stereo 


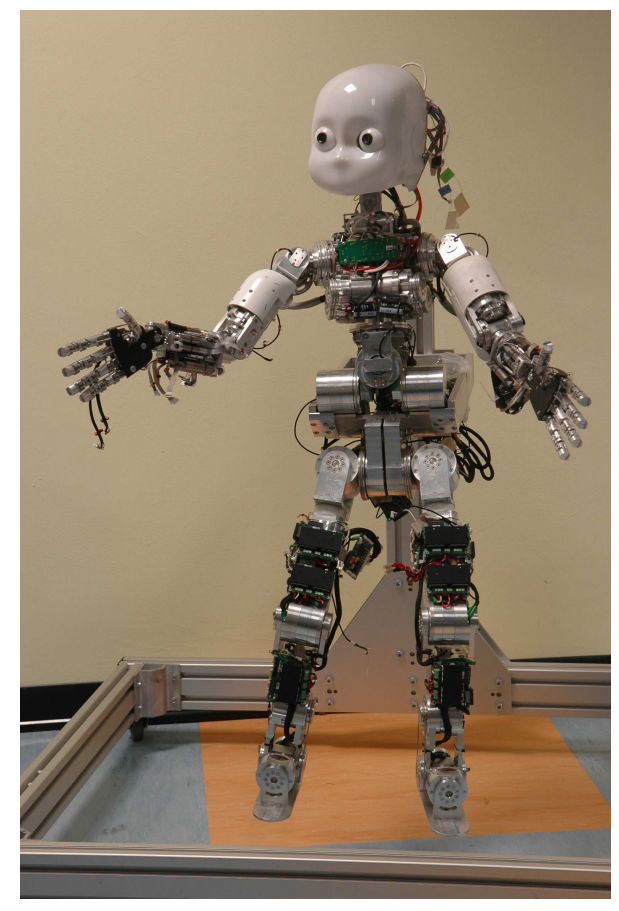

Fig. 1. The humanoid robot iCub. It has 53 controllable degrees of freedom that are driven by electrical motors. It is roughly $90 \mathrm{~cm}$ tall.

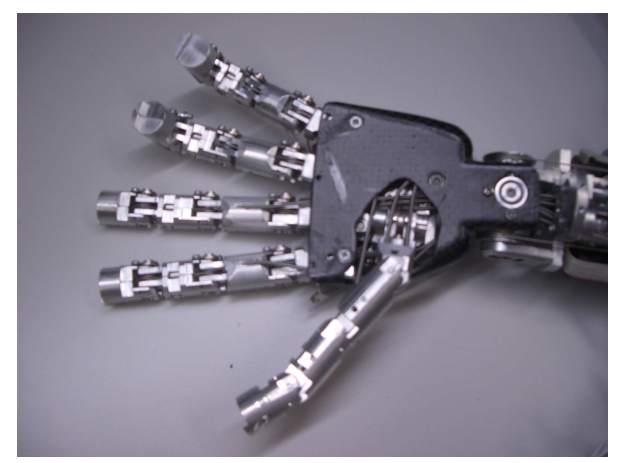

Fig. 2. A hand of the humanoid robot iCub. It has 9 controllable degrees of freedom, not including the wrist, and most of the actuators are located in the forearm.

vision system, microphones, acceleration sensors in the head and joint position sensors in all the joints.

The hand of the iCub is roughly $140 \mathrm{~mm}$ long and $60 \mathrm{~mm}$ wide. It has five underactuated fingers (see Figure 2) controlled by 9 motors. In practice two motors control independently the motion of the proximal and medial phalanges of the index finger; the most distal phalange is mechanically coupled with the medial one to naturally bend when the finger closes. A similar configuration is repeated for the middle finger. Ring and little fingers are coupled together and controlled only by one motor. The thumb is actuated by three motors: it can rotate around the palm and flex at the level of proximal and medial phalanges. Finally, another motor controls the abduction of the index, ring, medium and little finger.

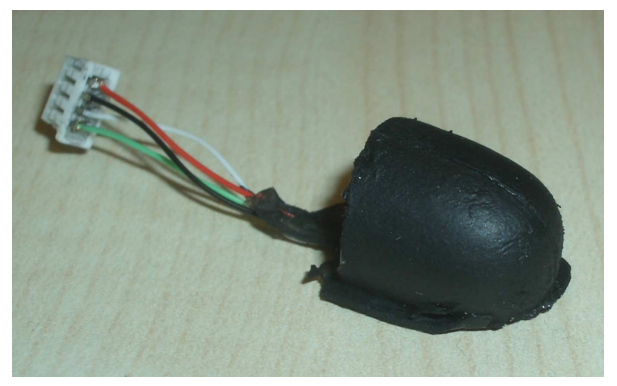

Fig. 3. A picture of the first version of the fingertip. The outer conductive layer is black because of a carbon black-silicone deposition made to create a flexible conductor. The fingertip includes a capacitive pressure sensor with 12 sensitive zones. The PCB with the electronics is included in the fingertip. Not only the outer layer, but also the inner core and the electrode structure can be made of highly flexible silicone, which makes the fingertip compliant.

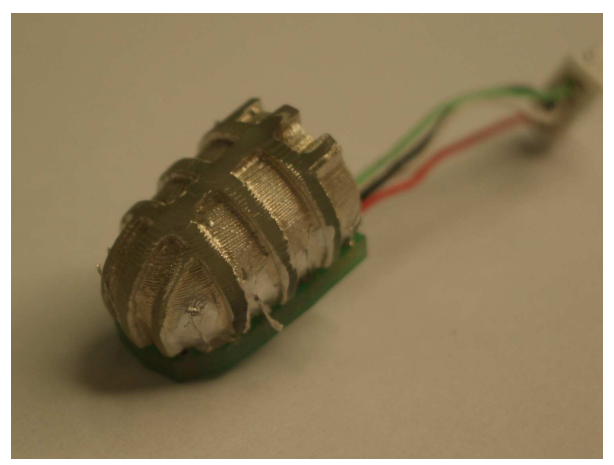

Fig. 4. A picture of the hard fingertip prototype without its outer layer. One can see the inner core and the PCB. The sensitive zones are made of conductive ink (silver).

\section{Description OF THE FINGERTIP}

Figure 4 and 3 show the prototype of the fingertip. It was designed to fit the avilable space on the hand. As a result it is $14.5 \mathrm{~mm}$ long and $13 \mathrm{~mm}$ wide (see Figure 7). The sensors are capacitive. In practice the fingertip is made of compliant and deformable silicone patches whose capacitance varies when pressure is applied at the surface. The capacitors surround the inner core of the fingertip, which is mounted on a small printed circuit board, as shown in Figure 5 and 6.

\section{A. Capacitive Pressure Sensor}

The transducer of the capacitive pressure consists of two conductive layers separated by a soft insulator made of silicone foam. The inner conductive layer is separated into 12 areas (see Figure 7), forming the taxels, acting as electrodes. When pressure is applied to the fingertip, the silicone foam gets compressed, and the capacitance between the inner and outer conductive layers changes as they get closer. The change of capacitance is taken as an estimation of the deformation of the silicone foam. This in turn is proportional to the pressure applied to the sensor.

The PCB with the electronics is included in the fingertip. It connects all the electrodes of the capacitive pressure transducer to an off-the-shelf capacitance to digital converter (CDC) (AD7147 from Analog Device in the present implementation), 


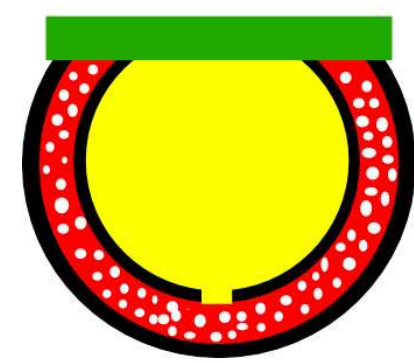

Fig. 5. A cross-section of the fingertip. The board is shown in green; the inner core, which can be either hard or soft, is yellow; the silicon foam is red; the conductive material black.

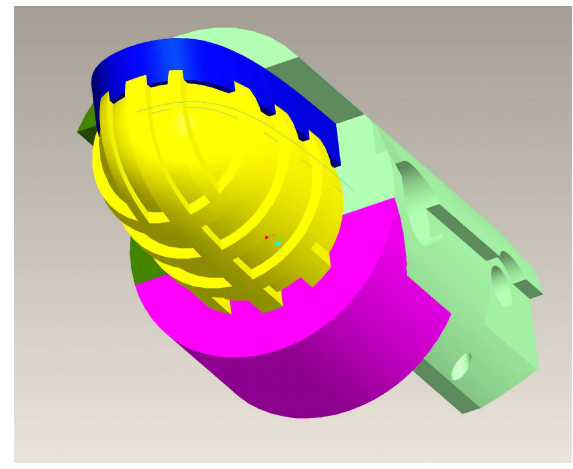

Fig. 6. A section of the internal structure of the fingertip: the inner core/electrodes support (yellow), the aluminum phalange (green), the PCB in blue and the cover silicone (only partially shown, in purple).

which is mounted on the PCB. The CDC is able to provide twelve 16 bits measurements of capacitance and send them, using serial bus communication, through a digital line. This is beneficial because it allows digitizing the signal as close as possible to the transducer, thus improving the signal to noise ratio, and, more importantly, it reduces the number of wires that travel from the fingertips to the hand. In this case, in fact, only four wires are necessary.

The data from all the CDCs of one hand is sent to a microcontroller unit, which is small enough to be included in the forearm of the iCub; in its current implementation it is $25 \mathrm{~mm}$ long and $17 \mathrm{~mm}$ wide. Each microcontroller unit can collect the measurements from up to $16 \mathrm{CDC}$ chips and send the data to a host using a CAN bus. The microcontroller unit is also used to program the CDC chips for different behaviors. The CDC chips are able to measure either all 12 taxels independently at $50 \mathrm{~Hz}$ or an average of the 12 taxels at a higher frequency (about $500 \mathrm{~Hz}$ ). Each fingertip can therefore be used adaptively to either detect contacts at high frequency with low accuracy or at a lower frequency with higher precision.

\section{B. Silicone Structure}

The silicone layer is overall only $2 \mathrm{~mm}$ thick in order to maintain good spatial resolution. We use the silicone foam (Soma Foama 15 Flexible Silicone Foam from Smooth-On ${ }^{1}$ ),

${ }^{1}$ www.smooth-on.com/

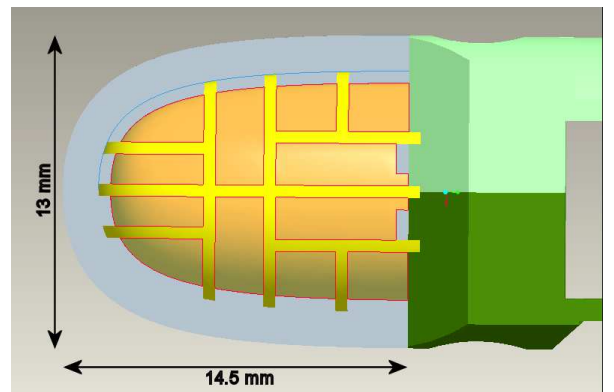

Fig. 7. Drawing of the inner core and the silicone foam from below. The electrodes, which act as the receptive fields, are highlighted in orange. The silicone foam is also visible, shaded in grey, to show to overall size of the fingertip.

because it compresses easily after the first contact. This makes the sensor very sensitive to light touch. The foam is filled with bubbles that when compressed enough make the whole structure somewhat stiffer. A stronger force is then necessary to compress the silicone even more. This non-linearity is useful to enhance the range of measurable forces. This principle has already been exploited in [11] although the scale of the air gaps was different. A similar property holds for the sensors described in [15]. The difference in this case is that the foam guarantees a homogeneous deformation that does not depend on the point of pressure.

Two versions of the fingertip have been built for comparison reasons, with hard and soft inner cores respectively. The later achieves maximal compliance without decreasing the sensor resolution: here also the inner core is made of soft silicone, namely Ecoflex 00-30 silicone from Smooth-On. We use it because of its high elasticity, toughness and shape stability (viscosity: $3000 \mathrm{cps}$, tear strenght: 38 pli, elongation at break: 900\%, tensile strength: $200 \mathrm{psi}$ ). Moreover, the 12 electrodes (receptive fields) have to be elastic and are therefore made from conductive silicone, the same material that is used for the outer electrically conductive layer.

The first tentative approach was based on conductive dyes to build generic tridimensional electrodes. Several off-the-shelf conductive dyes were tested but none proved effective. In practice the surface of the silicone layer deforms substantially causing the dyes electrodes to break. Electrical conductance was also too low for our application. More importantly, they were not fully adhesive to silicone. For these reasons, we used a mixture of silicone glue CAF-RTV1 from RhodiaSilicones and carbon-black particles Vulcan XC72 from Cabot. This material had excellent elasticity, adhesion to silicone, and sufficient conductivity (namely $10 \mathrm{k} \Omega$ ). We sprayed this material with the help of toluene.

Another problem was the realization of the 12 electrodes. This was mainly because the small size and three-dimensional round shape of the fingertip made it hard to deposit the conductive materials solely on the electrode areas. Most standard production methods are not suitable as they only work on twodimensional flat surfaces. In the prototype the electrodes were produced manually. The conductive material was deposited on 


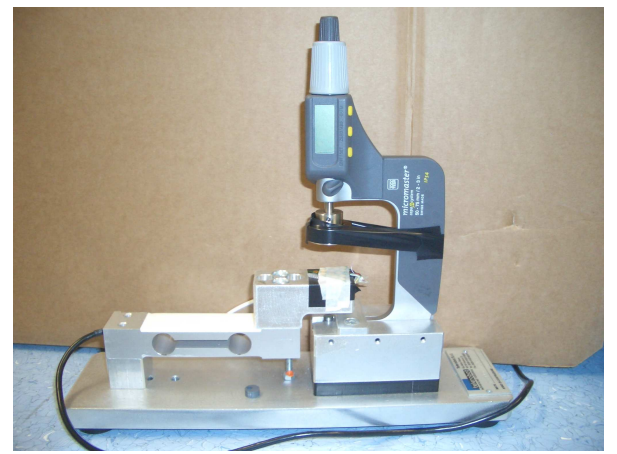

Fig. 8. Test setup with a load cell that can measure static forces. The off-center load cell (3 kg AL series, from Laumas) and the micrometer (TESA Micromaster IP54) are shown.

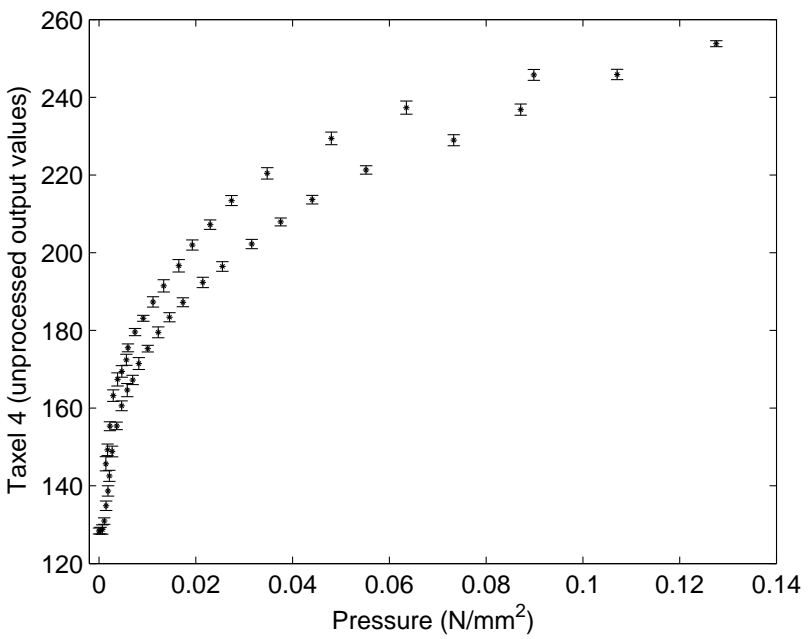

Fig. 9. The output of one of the receptive fields vs. the pressure as calculated from the measurements of the load cell. The pressure was increased and decreased is small steps, by moving a probe by means of a micrometer in steps of $0.1 \mathrm{~mm}$ toward and then away from the fingertip. For each step the average measurement of taxel 4 (the taxel placed directly below the probe) together with the standard deviation is shown. One can clearly see the nonlinear response of the capacitive sensor, with a higher sensitivity for lower pressures.

the whole surface of the inner core and was then removed only between the electrodes, using a small metal tool (small scissors and a file).

\section{EXPERIMENTAL RESULTS AND DISCUSSION}

Initial tests have been performed with the hard inner core prototype. We used an off-center load cell (3 $\mathrm{kg}$ AL series, from Laumas ${ }^{2}$ ) suitable to measure static forces. Pressure is applied with a micrometer (TESA Micromaster IP54) by moving a metal probe vertically against the sensor. The micrometer position can also be monitored. When it is moved downward, it applies pressure to the fingertip (see Figure 8). The contact area between the metal part and the fingertip is constant to about $18.6 \mathrm{~mm}^{2}$. The fingertip is mounted on the load cell,

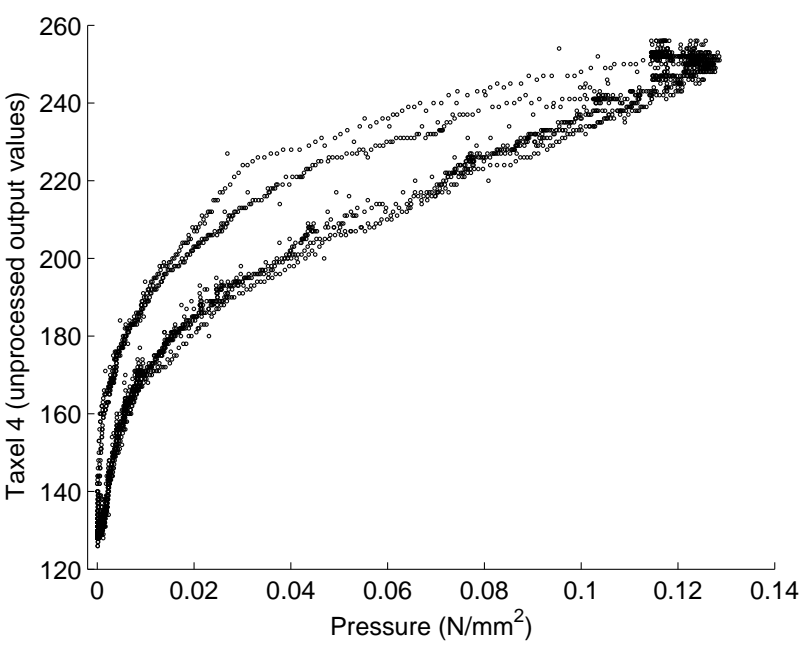

Fig. 10. The output of one of the receptive fields vs. the pressure as calculated from the measurements of the load cell. The pressure was increased and decreased uniformly. The process was repeated three times in a row.

the pressure is calculated as the ratio of the force over the contact area.

We moved the probe in small steps of $0.1 \mathrm{~mm}$ each, to cover the available range. In each step we collected data from the fingertip and the load cell for about 5 seconds and then manually changed the position of the probe. Figure 9 shows the output of the taxel immediatly below the probe (taxel 4) and the measured pressure. Figure 10 reports data collected when continuously increasing and decreasing the stimulus several times over the course of a couple of minutes. The figure shows how much the measurements change between repetitions. When increasing the pressure we could observe the nonlinear response predicted by the deformation of the silicone foam as discussed previously. This property was expected and it might be indeed useful allowing a wider dynamic range. While the sensor is particularly sensitive to very low pressures, we could measure pressure up to about $0.13 \mathrm{~N} / \mathrm{mm}^{2}$. Unfortunately, we could also clearly observe hysteresis, which is probably due to the relaxation of the silicone foam.

In a second set of experiments we used a system which applies periodic pressure to the fingertip against a load cell (PCB 208 C01 dynamic load cell ${ }^{3}$, see Figure 11). Stimulation is achieved by placing a vibrating probe against the fingertip which in turn lays against the load cell; this setup allows controlling the amplitude and the frequency of the vibration. The load cell measures the force between the probe and the surface of the fingertip. We compared the data obtained from taxel 4 of the fingertip and the force read from the load cell. Figure 12 reports the data we collected and it shows that the two are qualitatively similar (values are normalized to facilitate comparison). This plots also shows the dynamic response of the sensor, although more tests are required to

\footnotetext{
${ }^{3}$ www.pcb.com/techsupport/tech_force.php
} 


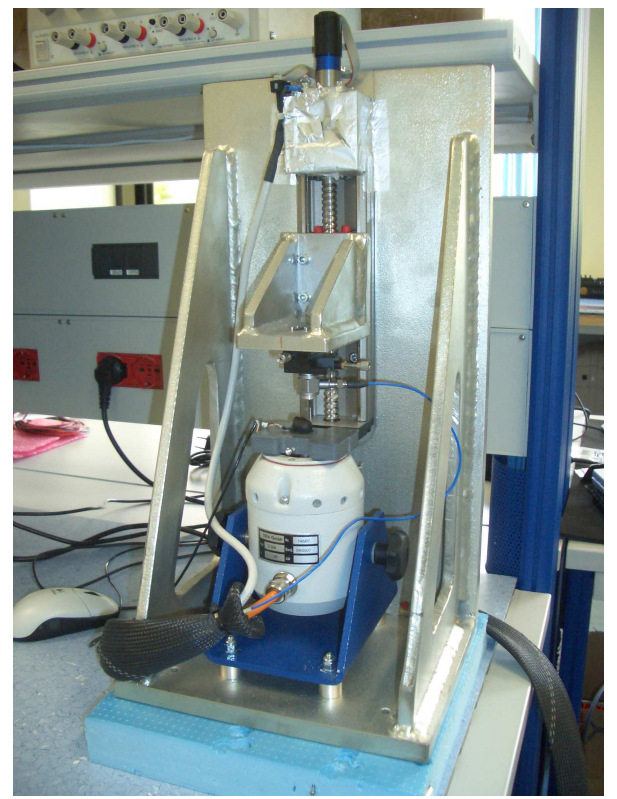

Fig. 11. Experimental setup with dynamic load cell used to measure the response of the sensor. Initial tests have been performed with a shaker moving the fingertip vertically with a sinusoidal profile. The data obtained from one of the receptive fields was compared to the force measured by a PCB 208 C01 dynamic load cell.

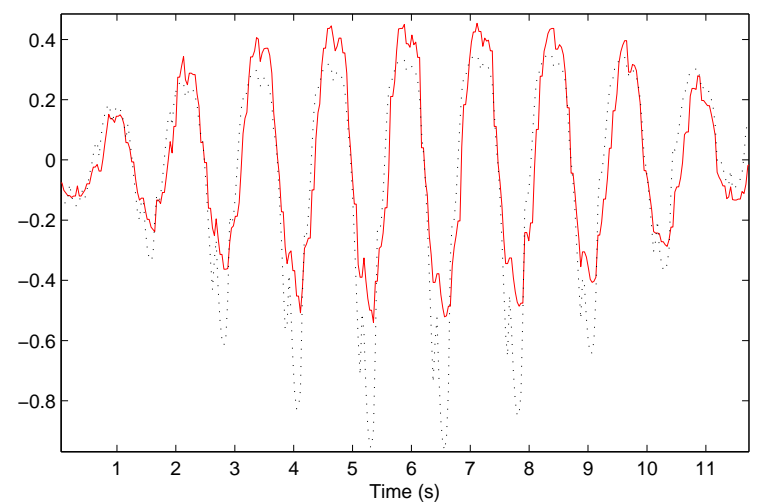

Fig. 12. The normalized output of the dynamic load cell (black, dashed) and the capacitive pressure sensor (red). The shaker moved the fingertip vertically with a sinusoidal profile, and simultaneously the amplitude was modulated sinusoidally. One can clearly see the similarities between the two measurements. The different amplitudes of the two signals stem solely from the different output ranges of the sensors.

properly measure its frequency response.

\section{CONCLUSiOns ANd Future WORK}

We presented the first version of a small fingertip for the humanoid iCub [17]. The design focused on the optimization of the size, spatial resolution and mechanical compliance of the sensor structure. The fingertip embeds the PCB with the electronics which performs signal conditioning and digitalization: this reduces the size of the fingertip and the number of wires required to connect it to the main robot electronic infrastructure. Initial experiments have been performed: while the initial results are promising, a number of tests remain to be done. In particular, a full-blown characterization of the sensor remains to be done including the frequency response of the single taxel and the cross-talk between taxels. Given the application, reliability is also to be considered.

The prototype has a certain number of issues that we started addressing in a second version:

- The PCB does not provide a connection to the ground layer and the position of the output connector is not compatible with the structural parts of the fingertip. This makes difficult a proper connection of the fingertip on the finger support. Clearly, this is an easy fix by moving the connectors to a more suitable location.

- The space on the PCB for the electrical connections to the 12 receptive fields is small and was done manually; the production of each fingertip is time consuming and errorprone. We are currently investigating various solutions to make the fabrication fully automatic by exploiting standard "pick and place" machines.

- The CDC chip and the receptive fields are on the same side of the PCB; this adds noise to the signal as the signal conditioning and the signal measurement influence each other. This can be fixed by redesigning the PCB.

Future developments include increasing the resolution of the sensor to 24 receptive fields (the size of the CDC chip is compatible with this hypothesis), and thus making smaller receptive fields.

\section{ACKNOWLEDGMENT}

This work is funded by the European Commissions Unit E5 Cognitive Systems, Interaction and Robotics, DirectorateGeneral Information Society, as part of the project IST-FP6004370 RobotCub: ROBotic Open-architecture Technology for Cognition, Understanding, and Behaviour and project ICTFP7-215843 Poeticon.

\section{REFERENCES}

[1] H. Nicholls and M. Lee Tactile sensing for mechatronics - a state of the art survey. Mechatronics 9, 1999.

[2] J. J. Gibson The theory of affordances. R. Shaw and J. Bransford (eds), Perceiving, acting, and knowing: Toward an ecological psychology. Hillsdale, NJ, USA: Erlbaum, pp 67-82, 1977.

[3] P. Cisek, Beyond the computer metaphor: Behaviour as interaction. Journal of Consciousness Studies, 6(11), 125-142, 1999.

[4] G. Metta and P. Fitzpatrick Better Vision Through Manipulation. C. G. Prince, Y. Demiris, Y. Marom, H. Kozima and C. Balkenius (eds), Proceedings of the Second International Workshop on Epigenetic Robotics: Modeling Cognitive Development in Robotic Systems 94, pp 97-104, 2002.

[5] T. M. Kubow and R. J. Full The role of the mechanical system in control: a hypothesis of self-stabilization in hexapedal runners. Philosophical transactions- Royal Society of London. Biological sciences, 354(1385), 849-861, 1999.

[6] M. Desmurget and S. Grafton Forward modeling allows feedback control for fast reaching movements. Trends in Cognitive Sciences, 4(11), 423431, 2000.

[7] R. Pfeifer and C. Scheier Understanding Intelligence. Cambridge, MA, USA/London, UK: MIT Press/Bradford Books, 1999.

[8] M. H. Dickinson, C. T. Farley, R. J. Full, M. A. R. Koehl, R. Kram and S. Lehman How animals move: an integrative view. Science, 288, 100-106, 2000. 
[9] F. Iida, G. Gomez and R. Pfeifer Exploiting body dynamics for controlling a running quadruped robot. In: Proceedings of the 12th International Conference on Advanced Robotics (ICAR 2005), 2005.

[10] R. Pfeifer, F. Iida and J. C. Bongard New robotics: Design principles for intelligent systems. Journal of Artificial Life, 11, 99-120, 2005.

[11] L. Jamone, G. Metta, F. Nori and G. Sandini James: A Humanoid Robot Acting over an Unstructured World. Proceedings of 6th IEEE-RAS International Conference on Humanoid Robots, 2006, Genoa, Italy.

[12] H. Liu, P. Meusel, N. Seitz, B. Willberg, G. Hirzinger, M. H. Jin, Y. W. Liu, R. Wei and Z. W. Xie The modular multisensory DLR-HIT-Hand. Mechanism and Machine Theory, Volume 42, Issue 5, Pages 612-625, 2007.

[13] T. Mouri, H. Kawasaki, K. Yoshikawa, J. Takai and S. Ito Anthropomorphic Robot Hand: Gifu Hand III. Proceedings of International Conference ICCAS, pp 1288-1293, 2002.

[14] P. Dario, C. Laschi, A. Menciassi, E. Guglielmelli, M. C. Carrozza, M. Zecca, L. Zollo, G. Teti, L. Beccai, F. Vecchi, S. Roccella $A$ Human-like Robotic Manipulation System Implementing Human Models of Sensory-Motor Coordination. Proceedings of 3th IEEE-RAS International Conference on Humanoid Robots, 2003, Karlsruhe, Germany.

[15] L. Natale and E. Torres-Jara A sensitive approach to grasping. In: Proceedings of the 6th International Conference on Epigenetic Robotics, 2006.

[16] Shadow Hand Company [Online] Shadow dexterous hand. http://www.shadowrobot.com/hand/, March 1, 2008

[17] N. G. Tsagarakis, G. Metta, G. Sandini, D. Vernon, R. Beira, F. Becchi, L. Righetti, J. Santos-Victor, A. J. Ijspeert, M. C. Carrozza, and D. $\mathrm{G}$. Caldwell $i C u b$ - the design and realization of an open humanoid platform for cognitive and neuroscience research. International Journal of Advanced Robotics, Vol. 21 No. 10, pp. 1151-75, Oct. 2007. 